Article

\title{
On the Resilience of Ant Algorithms. Experiment with Adapted MMAS on TSP
}

\author{
Elena Nechita ${ }^{1, *}$, Gloria Cerasela Crişan ${ }^{1}$, Laszlo Barna Iantovics ${ }^{2}$ (I) and Yitong Huang ${ }^{3}$ \\ 1 Department of Mathematics and Informatics, Vasile Alecsandri University of Bacău, 600115 Bacău, Romania; \\ ceraselacrisan@ub.ro \\ 2 Department of Electrical Engineering and Information Technology, George Emil Palade University of \\ Medicine, Pharmacy, Science and Technology of Târgu Mureş, 540139 Târgu Mureş, Romania; \\ barna.iantovics@umfst.ro \\ 3 Computer Science Department, Illinois Institute of Technology, Chicago, IL 60616, USA; \\ yhuang109@hawk.iit.edu \\ * Correspondence: enechita@ub.ro
}

Received: 30 March 2020; Accepted: 27 April 2020; Published: 9 May 2020

\begin{abstract}
This paper focuses on the resilience of a nature-inspired class of algorithms. The issues related to resilience fall under a very wide umbrella. The uncertainties that we face in the world require the need of resilient systems in all domains. Software resilience is certainly of critical importance, due to the presence of software applications which are embedded in numerous operational and strategic systems. For Ant Colony Optimization (ACO), one of the most successful heuristic methods inspired by the communication processes in entomology, performance and convergence issues have been intensively studied by the scientific community. Our approach addresses the resilience of MAX-MIN Ant System (MMAS), one of the most efficient ACO algorithms, when studied in relation with Traveling Salesman Problem (TSP). We introduce a set of parameters that allow the management of real-life situations, such as imprecise or missing data and disturbances in the regular computing process. Several metrics are involved, and a statistical analysis is performed. The resilience of the adapted MMAS is analyzed and discussed. A broad outline on future research directions is given in connection with new trends concerning the design of resilient systems.
\end{abstract}

Keywords: resilience; nature-inspired algorithm; Ant Colony Optimization; MAX-MIN Ant System; Traveling Salesman Problem

\section{Introduction}

In recent decades, the community of researchers from various domains has shown a growing interest towards systems resilience. Of course, systems of any kind are expected to meet requirements and maintain their operational characteristics as long as possible, while facing changing (sometimes unpredictable) conditions, environments and challenges. The concept of resilience has emerged for complex, dynamic systems and can be generally defined as "the capacity of a system to tolerate disturbances while retaining its structure and functions" [1]. The list of domains where systems resilience is important is long, and specific definitions have been provided: engineering [1], economics [2,3], environment [4,5], ecology [6,7], psychology and neurobiology [8-10], sociology [11]. In computer science, resilience has been defined mainly for networks [12-15] and large-scale distributed systems [16,17], security [18] and soft infrastructure systems [19]. The term resilience is derived from the Latin "resilire", with the meaning "springing back" or "jumping back up", which sends us to the views of resilience considering the recovery of a system after facing adverse situations.

Given the wide interest and importance of the concept, not only for researchers but for policymakers too, numerous and sometimes diverging interpretations and perceptions have been proposed for 
resilience. Several studies proposed conceptual and theoretical models for resilience, such as Cutter et al. [20], Jordan and Javernick-Will [21] for societal resilience, Scott [22], MacAskill and Guthrie [23] for disaster risk management or Chien et al. [24] for high-performance computing systems. A methodology to draw together resilience concepts from multiple disciplines can be found in Wright et al. [25].

Considerations on resilience, as it was defined and approached by researchers in various domains of human activity, are given in Section 2, which also bridges this concept with that of algorithmic performance. Section 3 overviews some basic ideas and characteristics of the ant algorithms and describes the tools: Traveling Salesman Problem (TSP) as test problem and MMAS to be adapted to the research goals. Section 4 points to the experimental settings. Section 5 presents the experimental outputs and their statistical analysis, while Section 6 concludes on the research results and gives some lines for further investigations.

\section{The Concept of Resilience and Some of Its Instances}

This section presents the concept of resilience as it evolved over the ages and across areas of human activity and research, with the intent to underline its main characteristics and emphasize both its essence and its multiple facets.

For physical and social systems, resilience is defined in Bruneau and Reinhorn [26] as consisting of the following properties: robustness (the ability of elements to withstand a given level of stress or demand without suffering degradation or loss function), redundancy (the extent to which the system's elements are substitutable), resourcefulness (the capacity to identify problems, establish priorities and mobilize resources when disruption conditions manifest), and rapidity (the capacity to meet priorities and achieve goals in a timely manner in order to recover functionality and avoid future disruption). Defining the concepts of vulnerability and resilience, Proag [27] differentiates between two broad forms of the last one: hard resilience-the direct strength of a structure when placed under pressure, and soft resilience-the ability of systems to absorb and recover from the impact of disruptive events without fundamental changes in function or structure.

The need for designing systems that allow for their evolvability has been recognized [28], since the majority of changes that a system is going to cope with are not foreseeable at the moment of its design. Technology refreshment programs have been proposed [29] to enhance systems capability for facing external sources of change.

Computer scientists have used the term "resilient" since 1976 and use it more and more often, sometimes as a synonym of fault-tolerant [30]. Avizienis et al. [31] define resilience as "the persistence of service delivery that can justifiably be trusted, when facing changes" or "the persistence of avoidance of failures that are unacceptably frequent or severe, when facing changes". Resilient software systems are needed in applications executed on distributed platforms (very often, with a dynamically changing topology), satisfying real-time requirements, interacting with real-world and managed externally by an authority. Approaching software resilience implies to model the functionality that the system provides, the way the system can provide that functionality and the deployment of constraints. Axelrod [32] notes the definition given in Marcus and Stern [33] for a resilient system: "one that can take a hit to a critical component and recover and come back for more in a known, bounded, and generally acceptable period of time" and presents the factors that may diminish software resiliency: complexity, interdependency and interconnectivity, globalization, hybridization, rapid change and reuse in different contexts.

In Chandra [34], an extensive study regarding the synergy between biology and systems resilience is given. The thesis focuses on extracting principles and heuristics useful for designing resilient engineering systems, based on the resilience seen in nature. Resilience attributes (adaptability, redundancy, scalability, self-organization, interoperability, dynamic learning) are identified in immune systems, colonies of social insects and ecosystems, and a domain-independent qualitative model of resilience is developed.

When talking about resilience, it is important to measure it. Enhancing the resilience of a system involves the understanding of the relationships that exist between the various components of that 
system. Although models for systems resilience can be useful, these should be interpreted on specific context and purpose [35] and it is important to be able to quantify the resilience of systems.

Measuring resilience naturally depends on the system being studied. While qualitative assessment reveals how bad a system can be disturbed, quantitative measures give estimates of performance [36]. Some general considerations on metrics for resilience are given in Ford et al. [18].

For the purposes of our work, which approaches TSP with a nature-inspired type of algorithm, we have used several metrics, further described in Section 4.3.

\section{Adaptation of an Ant Algorithm to Allow Disturbances Simulation}

During the last century, TSP was one of the most studied NP-hard problems in Combinatorial Optimization and Operations Research, mostly due to its applications in industry, transportation and logistics. The ant colony metaphor was easily adapted to TSP. Sections 3.1-3.4 present the foundations of the ant algorithmic approach, TSP as test problem and the particular Ant algorithm adapted for tackling real-world issues of TSP.

\subsection{An Efficient Nature-Inspired Class of Algorithms}

An ant colony is an example of a highly distributed natural, cooperative multiagent system. It is comprised of hundreds (or thousands) of completely autonomous simple agents (the ants) and is robust with respect to loss of individual agents and to changes in the environment [37]. The performance of the activities of the colony can be seen as highly effective [38] and are, in some cases, optimal or near-optimal.

In a colony, the scouts are the ants exploring the territory around the nest in search for food. At the beginning, a scout wanders and finds a food source only by chance. As a result, the route the scout ant follows from the nest to the food source displays a random pattern. Conversely, the route back is straight-lined and impregnated with pheromones, which are a very elaborated form of chemical communication [39]. The scout alerts its worker mates, who follow the pheromone trail to the food source. The number of ants using the trail is proportional to the available amount of food and, as long as the food source is not exhausted, the ants will reinforce the path while carrying their load back to the nest. This behavior determines higher and higher concentrations of pheromone on the shorter paths, as more and more ants are using them. On longer paths, the pheromone concentration decays over time.

Based on this ant foraging model, Dorigo et al. [40,41] have founded a new paradigm of evolutionary computation that has come to be known as Ant Colony Optimization (ACO) [42]. ACO is perhaps the most successful application inspired by ants-and perhaps by any insect society. There are three ideas from natural ant behavior that have been transferred to the artificial ant colony: the preference for paths with high pheromone level, the higher rate of growth of pheromone on shorter paths and the trail mediated communication among ants.

Since its development, researchers have applied ACO to a significant number of combinatorial optimization problems including the Traveling Salesman Problem (TSP), the Vehicle Routing Problem, graph coloring, allocation problems, swarm robotics, routing in telecommunication networks, constraint satisfaction and many other related issues.

\subsection{TSP as Test Problem}

TSP [43-45] was the first combinatorial optimization problem successfully solved with ACO [41]. We chose the Euclidean TSP as a support problem for the experiments on the resilience of ant algorithms implementations mostly due to its numerous real-life applications, most of which involve uncertainty. This characteristic is highly adequate for the aim of our study, namely, to analyze the ant algorithms capacity to "turn back" towards good solutions, even after significant interventions in the normal ACO execution process.

Given a map with $n$ sites and their pairwise distances, TSP requires finding one shortest cyclic tour through all the sites or, more formally, the least cost Hamiltonian cycle in a weighted graph. Formally, 
let $G=(N, A)$, with $N$ the set of nodes (locations) and $A$ the set of arcs fully connecting the nodes. Each arc $(i, j)$ is assigned the positive value $d(i, j)$ which is the distance between locations $i$ and $j$. TSP is the problem of finding the shortest closed tour visiting the $n=|N|$ nodes of $G$ exactly once.

TSP is a NP-hard problem [46]. However, polynomial time exact algorithms are available in particular cases [47]. Relaxation methods [48,49] have been used to develop Concorde [50], the best TSP exact solver at present. However, there are practical situations that could efficiently use results provided with approximate methods for TSP models [44,51-54]. Heuristic and metaheuristic approaches for TSP have also been intensively studied: local search [55-57], Lin-Kernigan [58], chained Lin-Kernigan methods [59], simulated annealing [60,61], genetic algorithms [62], and ant colony optimization (ACO) $[42,63]$.

\subsection{Implementations of Ant Algorithms for TSP}

The software package ACOTSP [64], freely available for both researchers and practitioners, includes implementations of ACO Algorithms for the symmetric TSP: Ant System, Elitist Ant System, MAX-MIN Ant System, Rank-based Ant System, Best-worst Ant System, and Ant Colony System. The performance of these implementations depends on instance dimension, parameter variation strategies, and particular improvements that may be operated upon (such as ants' specialization [65], human intervention in the ant loop [66], pheromone correction methods [67]) but is generally perceived by the scientific community as reasonably high. What makes these implementations very "usable" is their capacity to return as high-quality solutions as possible, independently of the allowed computation time $[68,69]$.

Ant System (AS) was the first ACO algorithm, proposed by Dorigo et al. in 1991 [70-72]. The algorithm of TSP solving with AS assumes a colony of $m$ artificial ants that operate on the graph $G$ and a pheromone matrix $\tau=\left\{\tau_{i j}\right\}$ for the construction of the solutions. At the beginning, $\tau_{i j}$ are set to the same initial, positive value. The $m$ ants are randomly deployed in the nodes of G. Within $t_{\max }$ iterations, the following repeat.

Each ant constructs a complete tour using Equation (1) below. If $t$ is the index of the current iteration, an ant $k$ currently at node $i$ chooses the node $j$ to move to (from the set $J_{k}(i)$ of the nodes not visited yet) by applying the following probabilistic transition rule:

$$
p_{i j}^{k}(t)=\left\{\begin{array}{cc}
\frac{\left[\tau_{i j}(t)\right]^{\alpha} \times\left[\eta_{i j}\right]^{\beta}}{\sum_{\epsilon \epsilon_{k}(i)}\left[\tau_{i l}(t)\right]^{\alpha} \times\left[\eta_{i l}\right]^{\beta}}, & \text { if } j \in J_{k}(i) \\
0, & \text { otherwise }
\end{array}\right.
$$

where $\eta_{i j}=1 / d(i, j)$ stands for the heuristic information called "visibility" of arc $(i, j)$, while $\tau_{i j}(t)$ represents the "desirability" that the arc $(i, j)$ belongs to the tour of the ant $k$. This desirability reflects the experience acquired by ants during the problem-solving and models a learning process. Parameters $\alpha$ and $\beta$ set the balance between the influence of the pheromone level and that of the heuristic information. Pheromone update is applied according to Equation (2) for all ants $k$ and all $\operatorname{arcs}(i, j)$ :

$$
\tau_{i j}^{k}(t+1)=(1-\rho) \cdot \tau_{i j}^{k}(t)+\Delta \tau_{i j}^{k}(t)
$$

where $\rho \in(0,1]$ represents the proportion of the old pheromone quantity that is carried over to the next iteration of the algorithm and the added quantity of pheromone as defined in Equation (3), with $T^{k}(t)$ the tour built by ant $k$ and $L^{k}(t)$ its length:

$$
\Delta \tau_{i j}^{k}(t)=\left\{\begin{array}{cc}
1 / L^{k}(t), & \text { if }(i, j) \in T^{k}(t) \\
0, & \text { otherwise }
\end{array}\right.
$$

For our experimental study concerning the resilience of the ant algorithms, we have chosen the MAX-MIN Ant System (MMAS) proposed by Stützle and Hoos [73] to be modified according to the 
purpose of our research. Although the focus is on resilience and not on performance, it is important to note that MMAS was designed as an algorithmic variant to achieve better performance than the ant system, from which MMAS originates.

MMAS is the same as AS except that the pheromone trails are updated offline: only the arcs which were used by the best ant in the current iteration receive additional quantities of pheromones. Also, the pheromone trail values are placed in an interval $\left[\tau_{\min }, \tau_{\max }\right]$ and the trails are initialized to their maximum value $\tau_{\max }$.

Moreover, MMAS calls the 3-opt local search procedure [58] at the end of the tour completion for every ant, thus being a hybrid method, which integrates both constructive and heuristic local-search.

\subsection{New Parameters for MMAS}

Let MMAS(epoch, p_nod_ch,p_inten, ampl) be the MAX-MIN Ant System enriched with a set of parameters, which are described below. The role of the parameters resides in introducing various disturbances in the algorithmic loop which determines the solution construction.

The idea is to alter the pheromone matrix periodically. This process allow us to simulate features such as uncertainty and dynamicity in the TSP instances to be approached with MMAS(epoch, p_nod_ch, p_inten, ampl). These features reside in numerous real-world problems that are modelled with TSP, which justifies our approach. It assumes that the number of iterations when the system works without intervention defines the parameter epoch. At the beginning of MMAS(epoch,p_nod_ch, p_inten, ampl), the algorithm works as presented in Section 3.3. At the end of the first period defined by epoch (after a number of iterations equal to epoch), the values in the pheromone matrix are changed non-deterministically: the degree of perturbation is introduced by means of the parameters $p \_n o d \_c h$ - the percentage of the nodes to suffer modifications, and $p \_$inten — the percentage of arcs incident to the chosen nodes, arcs whose pheromone load are modified. The parameter ampl gives the amplitude of the modification, setting an interval of length $2 * a m p l * o l d \_v a l u e$, centred in the old pheromone value. The new pheromone value is randomly generated within this interval. The process repeats all along the algorithmic loop of MMAS. The above parameters' identifiers head the data columns in the files available at [74].

This design of MMAS was also used by Crişan et al. in [75] in order to perform an ant-based system analysis on TSP under real-world settings, when comparing it with other hybrid optimization techniques. The feature introduced by these new parameters is the uncertainty that manifests in the real world. This alteration of the pheromones may simulate missing or imprecise data, which frequently happens in real-world systems that include TSP solvers.

\section{Experimental Settings}

Our experiment concerning the ant algorithms resilience was performed with the modified MMAS(epoch, p_nod_ch,p_inten, ampl) and three TSP instances. Specific details regarding instances, software, values for its parameters and organization of the output data are given below.

\subsection{TSP Test Instances}

The instances chosen for tests in our experiment are from TSPLIB [76,77], the well-known library of sample instances for combinatorial optimization problems. The problem names (which also include the number of nodes of the corresponding graph) are d2103, fl3795 and rl1889. For all three, the 2-dimensional Euclidean distances between nodes are rounded up to the next integer. The optimum tour lengths are known to be 80,450, 28,722 and 316,536, respectively.

$d 2103$ is one of the instances proposed by Reinelt [76], coming from the practical problem of drilling holes in printed circuit boards. The drilling problem can be approached as a sequence of TSP instances, one for each hole diameter [78]. fl3795 is another drilling instance, with a high degree of difficulty [79] given by the placement of the nodes in very particular clusters. The last instance, $r l 1889$, is one of the examples drawn by Reinelt [76] from geographic problems featuring locations of cities on maps. 


\subsection{Parameters Values and Ant Implementation}

The Ant implementation that we propose is synthetically described by the following algorithmic scheme. The skeleton is according to [73], and the new parameters epoch, p_nod_ch, p_inten, ampl are introduced.

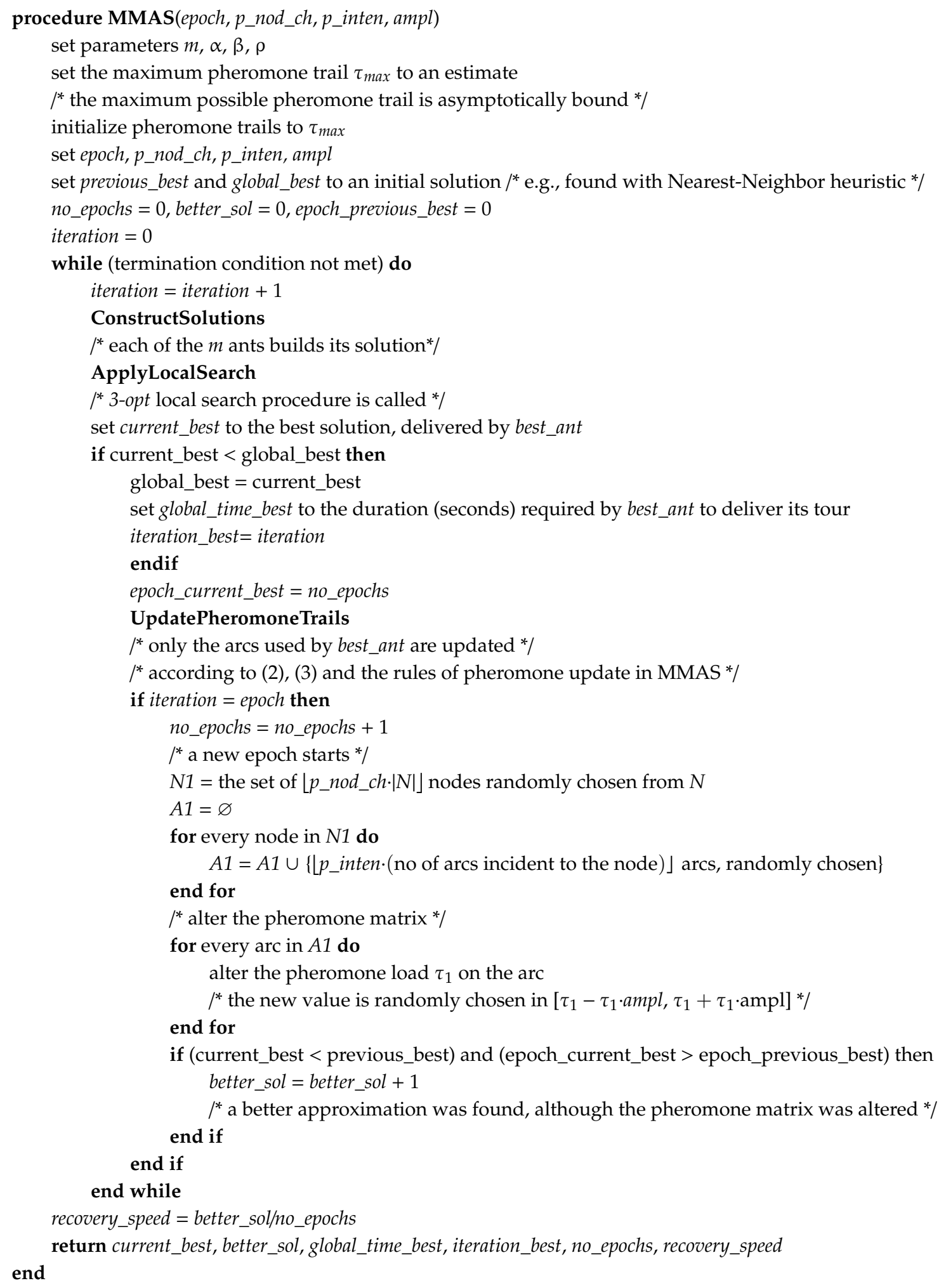

The original parameters used for MMAS(epoch, $\left.p \_n o d \_c h, p \_i n t e n, a m p l\right)$ in our experiments are: number of ants $m=25 ; \alpha=1 ; \beta=2 ; \rho=0.2$. 
The values for the new parameters are as follows: epoch $\in\{20,40,60\} ;$ _nnod_ch $\in\{20,40,100\}$; p_inten $\in\{50,100\}$, ampl $\in\{0,10,20,30,40\}$.

For each of the three instances, all the 3.3.2.5 $=90$ variants of MMAS, generated by the possible combinations of the parameter values, were executed. For each variant, ten trials have been performed in order to report a line in the result files, following the guidelines proposed by Birattari et al. [80]. One core (of a dual-core processor at $2.5 \mathrm{GHz}$ frequency and $250 \mathrm{~GB}$ RAM) was assigned only for the execution of MMAS software.

\subsection{Recorded Data}

For every TSP instance, the following execution outputs and statistics were recorded. These data are stored in a file with the same identifier as the name of the instance, available for download at [74].

- better_solutions: number of times when the application manages to find a better approximation of the optimum tour, although the information stored on arcs (the pheromone quantities) was disturbed (averaged over ten trials).

- number of epochs: how many times during the execution the information on the arcs was disturbed (on average, in ten trials). During an epoch, the pheromones evolve according to Equations (2) and (3).

- average-best: this is the main metric based on which we study the algorithm resilience. It represents the approximation of the TSP instance solution, as delivered by the modified ant algorithm MMAS (also averaged over ten trials).

- recovery speed: is the ratio between better_solutions and number of epochs. It gives a measure of the recovering capacity, showing how many times (on average, in ten trials) average-best improves during an epoch.

- average-iterations: is the average of the ten iteration numbers that found the best solution.

- stddev-best: standard deviation for the ten best solutions delivered by the ten trials.

- stddev-iterations: standard deviation for the ten iterations when the best solution was found.

- best try: the best solution in ten trials.

- worst try: the worst solution in ten trials.

- avg. time-best: average of the times for finding the best solution in ten trials.

- stddev.time best: standard deviation for the time durations needed to find the best solution, for the ten trials.

- distance-to-optimum: is the difference between the (average) solution found by the ant algorithm (average-best) and the optimum solution.

- relative-error: distance-to-optimum/optimum tour length $* 100$.

Using the instance $d 2013$ and the following values for the new parameters: epoch $=20, p \_n o d \_c h=20$, p_inten $=50, a m p l=10$ (which corresponds to line 22 in the data file $d 2013$ ), the adapted MMAS works as described below.

In a trial of $\operatorname{MMAS}(20,20,50,10)$, after every 20 iterations, for $\lfloor 20 / 100 \cdot 2103\rfloor=420$ nodes randomly chosen, the pheromone load is modified for half of the arcs (also randomly chosen) of the selected nodes. The number of arcs in this situation is $420 \cdot[50 / 100 \cdot 2102\rfloor=441,420$. For every arc, the new pheromone value is randomly chosen in an interval of length $2 \cdot(10 / 100) \cdot v$, centred in $v$, which is the old pheromone value. Under these settings, on average in 10 trials, we obtained the following outcome:

- number of epochs $=516$. We obtained 516 series of 20 iterations and, starting with the second one, the pheromone alteration takes place as described above.

- better_solutions $=246$, that is, although the pheromones are altered at every 20 iterations, the algorithm improves the solution, getting closer to the optimum, 246 times.

- average-best $=80,555.5$. This last approximation delivered by the algorithm is only at $105.5=$ distance-to-optimum (units) far from the known optimum, which is 80,450 . We notice 
that 105.5 represents $0.13 \%$ (= the value of the relative-error) from the optimum. The algorithm succeeded to provide a solution that is only $0.13 \%$ worse than the optimum, which is a very good outcome, given the repeated intervention into the solution construction mechanism. In terms of resilience, we can assess that this is high for $\operatorname{MMAS}(20,20,50,10)$.

- $\quad$ stddev-best $=54.64$. The solutions delivered by the algorithm are spread around the average-best $=$ $80,555.5$ with a standard deviation of 54.64 . The best solution among the ten collected in ten trials is best try $=80,476$ and the worst is worst try $=80,638$.

- average-iterations $=633.5$, that is, the average of the iteration numbers when the ten best solutions were delivered. The standard deviation of these ten values is stddev-iterations $=249.93$, quite a big value, showing that the best solution can be found early in the iterative process, or after a significant number of iterations.

- avg. time-best $=36.81 \mathrm{~s}$. This is the average value of the execution durations (measured in seconds) of the 10 trials. Given the computer system configuration that the experiment was processed on, the result is very good. The standard deviation for the execution times of the ten trials is stddev.time best $=13$.

Finally, recovery speed $=0.4767$ for this example. The value shows that, for instance $d 2103$, $\operatorname{MMAS}(20,20,50,10)$ improved the solution, on average, 0.4767 times per epoch.

\section{Results and Statistical Analysis}

\subsection{Metrics for TSP Resilience Assessment with Adapted MMAS}

According to Jackson [81], "System resilience is the ability of [...] systems to mitigate the severity and likelihood of failures and loses, to adapt to changing conditions, and to respond appropriately after the fact".

Researchers in systems resilience point that resilience is not a 1-dimensional quantity [18]. Moreover, a shift from robustness-centered design to principles of more flexible and adaptive design has been noticed [33]. This approach includes the following ideas: composing the measures of different aspects in order to reason about resilience; the metrics should be particular for a specific perturbation; the metrics should be dependent on the system boundaries; the customer requirements drive the metrics for resilience; take into consideration other aspects except for the system output. There is no universal way to combine the particular metrics into a global one, but the assessment process is specific to the synergy of all considered aspects.

When transferring the above considerations to the transportation field, resilience could be seen as the ability of transportation systems to react to unexpected disturbance. This can be seen at the individual level, when transportation needs are satisfied for each customer, as well as at the community level-such as in emergency state or special events, when this perspective comes first. Transportation systems diversity (which includes multiple modes, routes, and system components) and critical information related to collection and distribution are key elements for resilience [82]. Both these features can be modelled in ant algorithms [83].

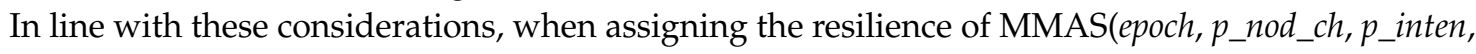
ampl) we chose to consider two kind of metrics:

- Time-performance metrics: better_solutions, number of epochs, average-iterations, stddev-iterations, avg. time-best, stdev.time best and recovery speed. All these data give us a feedback on how fast the altered applications work.

- Path-performance metrics: average-best (which is the objective function) and stddev-best, best try and worst try, three measures indicating how good the altered applications still perform.

Sections 5.2-5.4 present both a brief descriptive analysis (which can be used for a qualitative assessment of the MMAS applications resilience) and a statistical one, based on the results of the experiment, for each of the three TSP instances. The files presenting extensive statistical analysis are available at [74]. 
For the descriptive analysis, only a part of the data from the files $d 2103, f l 3795$ and $r l 1889$ was extracted in Tables 1, 4 and 7, respectively. The tables present the results obtained for the metrics recovery speed and average-best, reported for the highest value of ampl: $40 \%$. That is, if $\omega$ is a pheromone value on a selected arc (according to $p \_n o d \_c h$ and $p \_$inten) then the altered pheromone value randomly falls within the interval $[0.6 \cdot \omega, 1.40 \cdot \omega]$. These 18 variants of MMAS (of all 90) are those simulating the most substantial disturbances in the ACO constructive process of the approximate solution. Moreover, each table includes the lines for which the smallest and the highest values for the relative-error have been registered in all the 90 MMAS variants. For example, in Table 1, these correspond to executions with $a m p l=0$ (which is the original MMAS, with no altered pheromones).

Table 1. Parameters and metrics for $d 2103$, with $a m p l=40$.

\begin{tabular}{crrrrrr}
\hline Line & p_nod_ch & \multicolumn{1}{c}{$\boldsymbol{p}$ _inten } & epoch & recovery speed & average-best & relative-error \\
\hline 1 & 20 & 50 & 20 & 0.4487 & $80,595.2$ & 0.18 \\
2 & 20 & 50 & 40 & 1.1479 & $80,554.3$ & 0.13 \\
3 & 20 & 50 & 60 & 1.0877 & $80,546.1$ & 0.12 \\
4 & 20 & 100 & 20 & 0.5082 & $80,521.6$ & 0.091 \\
5 & 20 & 100 & 20 & 0.7103 & $80,593.8$ & 0.18 \\
6 & 20 & 100 & 40 & 0.8844 & $80,602.9$ & 0.19 \\
7 & 20 & 100 & 60 & 1.0838 & $80,596.2$ & 0.18 \\
8 & 40 & 50 & 20 & 0.4192 & $80,606.0$ & 0.19 \\
9 & 40 & 50 & 40 & 0.9000 & $80,590.1$ & 0.17 \\
10 & 40 & 50 & 60 & 1.0904 & $80,546.4$ & 0.12 \\
11 & 40 & 100 & 20 & 0.6965 & $80,616.7$ & 0.21 \\
12 & 40 & 100 & 40 & 0.8670 & $81,092.4$ & $0.80^{2}$ \\
13 & 40 & 100 & 40 & 0.9945 & $80,564.2$ & 0.14 \\
14 & 40 & 100 & 60 & 1.3642 & $80,529.4$ & 0.10 \\
15 & 100 & 50 & 20 & 0.5019 & $80,537.9$ & 0.11 \\
16 & 100 & 50 & 40 & 0.8445 & $80,587.0$ & 0.17 \\
17 & 100 & 50 & 60 & 1.2384 & $80,557.8$ & 0.13 \\
18 & 100 & 100 & 20 & 0.4173 & $80,583.8$ & 0.17 \\
19 & 100 & 100 & 40 & 1.1618 & $80,647.5$ & 0.25 \\
20 & 100 & 100 & 60 & 1.2051 & $80,561.8$ & 0.14 \\
\hline & & & 1,2 Values for ampl=0. & &
\end{tabular}

For the statistical analysis, two directions are set. With epoch, $p \_n o d \_c h, p \_i n t e n$ and ampl as factors, the following are studied:

- The way the time-performance metrics depend on the factors and how these are inter-correlated.

- The way the path-performance metrics depend on the factors and how these are inter-correlated.

The material displaying the results of the two approaches is quite extensive, and thus we present in Tables 2, 3 (for 2 2103), 5, 6 (for fl3795), and 8, 9 (for rl1889), the regression models for the metrics average-best and recovery speed only. The factors for the non-zero values of the categorical variable ampl (the amplitude of the pheromone alteration) are ampl10\%, ampl20\%, ampl30\%, and ampl40\%.

The statistical analysis was performed with the free software environment for statistical computing and graphics $\mathrm{R}$, version 3.4.4.

\subsection{Discussion on Behaviour of Adapted MMAS with TSP Instance d2103}

The length of the optimum solution for the TSP instance $d 2103$ is 80,450 . As data in Table 1 display in all lines except no. 4 and no. 12 , the relative error for the 18 variants of MMAS with $a m p l=40$ is placed in the interval [0.1,0.25], corresponding to an absolute error (distance-to-optimum in the file d2013) in the interval [82.1, 197.5]. We note that the minimum but also the maximum relative-error values (in lines 4 and 12) correspond to MMAS with ampl $=0$.

For the original MMAS, recovery speed $\in[0.4934,1.6154]$ (see file 22013 ), while for the applications corresponding to data in Table 1 (with ampl $=40$ ), recovery speed $\in[0.4173,1.3642]$ and only three values are smaller than the minimum recovery speed for MMAS with ampl $=0$. These findings show that 
$\operatorname{MMAS}(*, * *, 40)$ continue to behave normally, in the sense of building efficient solutions for the instance under consideration, successfully recovering after pheromone alterations.

The tableau depicted by the metrics average-best and recovery speed allows us to qualitatively assess the resilience of the adapted MMAS applications (according to the qualitative model in [34]) as High.

The regression model for the metric average-best, for instance $d 2103$, follows in Table 2.

Table 2. Regression model for average-best, instance $d 2103$.

\begin{tabular}{|c|c|c|c|c|}
\hline \multicolumn{5}{|c|}{ Dependent Variable: average-best } \\
\hline \multicolumn{5}{|c|}{ Method: Least Squares } \\
\hline \multicolumn{5}{|l|}{ Residuals: } \\
\hline Min & $1 Q$ & Median & $3 Q$ & $\operatorname{Max}$ \\
\hline-149.58 & -23.76 & -6.34 & 14.61 & 491.21 \\
\hline \multicolumn{5}{|l|}{ Coefficients: } \\
\hline & Estimate & Std. Error & t value & $\operatorname{Pr}(>|t|)$ \\
\hline (Intercept) & $8.058 \times 10^{+04}$ & $3.199 \times 10^{+01}$ & 2518.882 & $<2 \times 10^{-16 * * *}$ \\
\hline p_nod_ch & $-2.363 \times 10^{-02}$ & $1.986 \times 10^{-01}$ & -0.119 & 0.906 \\
\hline p_inten & $1.508 \times 10^{-01}$ & $2.700 \times 10^{-01}$ & 0.558 & 0.578 \\
\hline epoch & $6.450 \times 10^{-02}$ & $4.134 \times 10^{-01}$ & 0.156 & 0.876 \\
\hline ampl10\% & $-1.956 \times 10^{+01}$ & $2.135 \times 10^{+01}$ & -0.916 & 0.362 \\
\hline ampl $20 \%$ & $-2.938 \times 10^{+01}$ & $2.135 \times 10^{+01}$ & -1.377 & 0.172 \\
\hline ampl30\% & $-2.251 \times 10^{+01}$ & $2.135 \times 10^{+01}$ & -1.054 & 0.295 \\
\hline ampl40\% & $-1.838 \times 10^{+01}$ & $2.135 \times 10^{+01}$ & -0.861 & 0.392 \\
\hline \multicolumn{5}{|c|}{ Signif. codes: $0^{\star * * * \prime} 0.001^{\star * * \prime} 0.01^{\star * \prime} 0.05^{\prime \prime} .0 .1^{\prime \prime} 1$} \\
\hline \multicolumn{5}{|c|}{ Residual standard error: 64.04 on 82 degrees of freedom } \\
\hline \multicolumn{5}{|c|}{ Multiple R-squared: 0.02891, Adjusted R-squared: -0.05398} \\
\hline \multicolumn{5}{|c|}{ F-statistic: 0.3488 on 7 and $82 \mathrm{DF}, p$-value: 0.9287} \\
\hline
\end{tabular}

The adjusted low value of the R-Squared index shows that the linear regression model is bad. There is no relation between average-best and the predictors. The interpretation is that average-best is not influenced by the perturbations introduced by the parameters epoch, p_nod_ch, p_inten, ampl.

The regression model for recovery speed follows in Table 3.

Table 3. Regression model for recovery speed, instance $d 2103$.

\begin{tabular}{|c|c|c|c|c|}
\hline \multicolumn{5}{|c|}{ Dependent Variable: recovery speed } \\
\hline \multicolumn{5}{|c|}{ Method: Least Squares } \\
\hline \multicolumn{5}{|l|}{ Residuals: } \\
\hline Min & 1Q & Median & $3 Q$ & Max \\
\hline-0.31815 & -0.11957 & -0.01764 & 0.11520 & 0.54237 \\
\hline \multicolumn{5}{|l|}{ Coefficients: } \\
\hline & Estimate & Std. Error & $\mathrm{t}$ value & $\operatorname{Pr}(>|t|)$ \\
\hline (Intercept) & 0.0103022 & 0.0963029 & 0.107 & 0.91507 \\
\hline p_nod_ch & -0.0003021 & 0.0005978 & -0.505 & 0.61466 \\
\hline p_inten & -0.0010120 & 0.0008128 & -1.245 & 0.21665 \\
\hline epoch & 0.0235334 & 0.0012443 & 18.913 & $<2 \times 10^{-16 * * *}$ \\
\hline ampl10\% & 0.1944056 & 0.0642569 & 3.025 & $0.00332 * *$ \\
\hline ampl20\% & 0.1923444 & 0.0642569 & 2.993 & $0.00365 * *$ \\
\hline ampl30\% & 0.1871389 & 0.0642569 & 2.912 & $0.00462 * *$ \\
\hline ampl40\% & 0.0401833 & 0.0642569 & 0.625 & 0.53348 \\
\hline \multicolumn{5}{|c|}{ Signif. codes: $0^{\star * * * \prime} 0.001^{\star * * \prime} 0.01^{\star * \prime} 0.05^{\prime} !^{\prime} 0.1^{\prime \prime} 1$} \\
\hline \multicolumn{5}{|c|}{ Residual standard error: 0.1928 on 82 degrees of freedom } \\
\hline \multicolumn{5}{|c|}{ Multiple R-squared: 0.8213 , Adjusted R-squared: 0.8061} \\
\hline \multicolumn{5}{|c|}{ F-statistic: 53.85 on 7 and $82 \mathrm{DF}, p$-value: $<2.2 \times 10^{-16}$} \\
\hline
\end{tabular}

The adjusted R-Squared index shows that this linear regression model is good: based on the values of epoch, p_nod_ch, p_inten, and ampl, we could explain $80.61 \%$ of the variance of the recovery 
speed. The parameters $p \_n o d \_c h$ and $p \_$inten are negatively correlated with recovery speed, while epoch and ampl are positively correlated to it. The $p$ values show that the factors epoch and ampl are statistically significant in the model. Therefore, the recovery speed is influenced by the values of epoch and ampl.

In Figure 1, the correlogram between the other time-performance metrics is displayed. Positive correlations are displayed in blue and negative correlations in red. Colour intensity and the size of the circles are proportional to the correlation coefficients. In the right side of the correlogram, the legend colour shows the correlation coefficients and the corresponding colours.

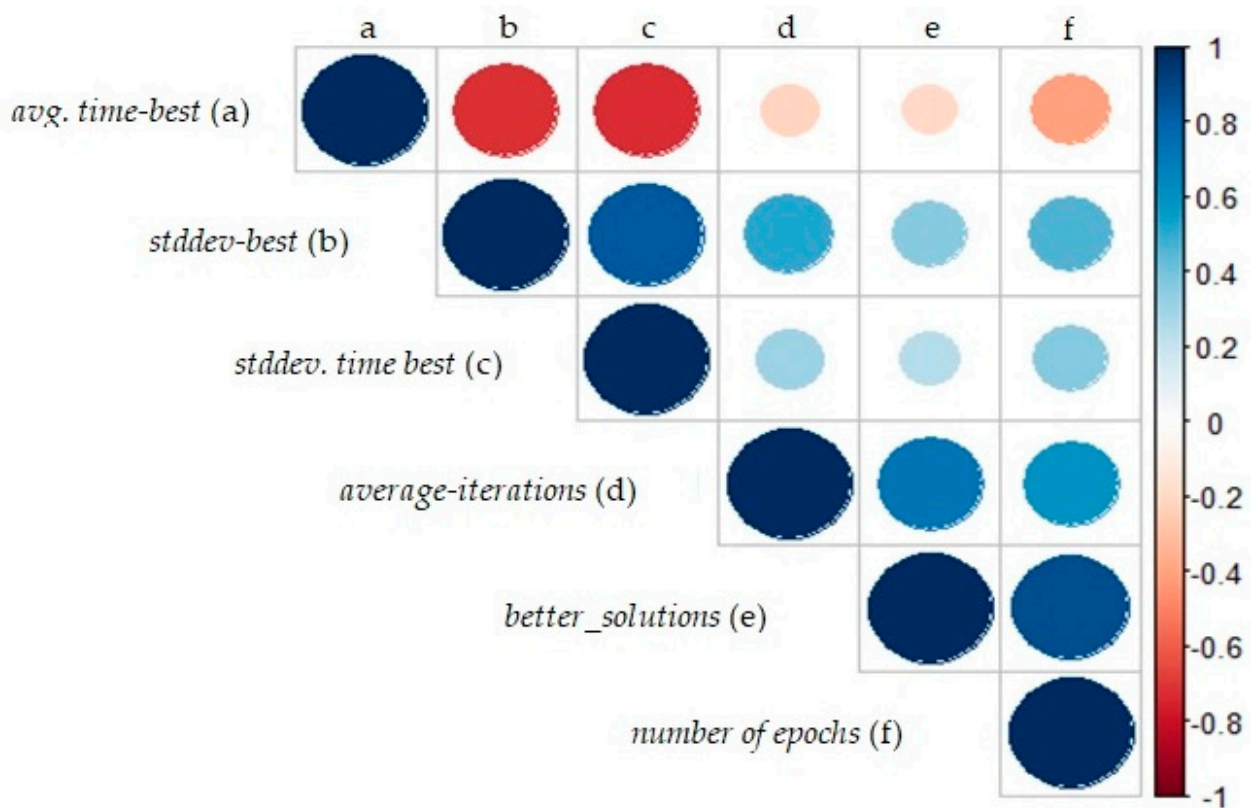

Figure 1. Correlogram of the time-performance metrics: avg.time-best, stddev-best, stddev.time best, average-iterations, better_solutions and number of epochs for $\mathrm{d} 2103$.

\subsection{Discussion on Behaviour of Adapted MMAS with TSP Instance fl3795}

The optimum for TSP instance $f l 3795$ is 28,722 . As data in Table 4 (except line no. 9) display, the relative error for the 18 variants of MMAS with ampl $=40$ is placed in the interval [3.84, 4.72], corresponding to an absolute error (distance-to-optimum in the file fl3795) in the interval [1101.5, 1354.3]. For this instance, the relative error is higher than in the case of the instance $d 2103$, but it can still be appreciated as small. We note that the minimum value for the relative-error is obtained for MMAS with ampl=10 (3.42, in line 9). 
Table 4. Parameters and metrics for $f l 3795$, with ampl $=40$.

\begin{tabular}{|c|c|c|c|c|c|c|}
\hline Line & p_nod_ch & p_inten & epoch & recovery speed & average-best & relative-error \\
\hline 1 & 20 & 50 & 20 & 0.4651 & $29,853.4$ & 3.94 \\
\hline 2 & 20 & 50 & 40 & 0.5946 & $29,895.6$ & 4.09 \\
\hline 3 & 20 & 50 & 60 & 0.5500 & $29,969.7$ & 4.34 \\
\hline 4 & 20 & 100 & 20 & 0.4414 & $29,848.0$ & 3.92 \\
\hline 5 & 20 & 100 & 40 & 0.6176 & $29,904.7$ & 4.12 \\
\hline 6 & 20 & 100 & 60 & 0.6087 & $29,875.0$ & 4.01 \\
\hline 7 & 40 & 50 & 20 & 0.4538 & $29,868.3$ & 3.99 \\
\hline 8 & 40 & 50 & 40 & 0.7027 & $29,960.9$ & 4.31 \\
\hline 9 & 40 & 50 & 60 & 0.6750 & $29,705.0$ & $3.42^{1}$ \\
\hline 10 & 40 & 50 & 60 & 0.5667 & $29,904.5$ & 4.12 \\
\hline 11 & 40 & 100 & 20 & 0.5299 & $29,823.5$ & 3.84 \\
\hline 12 & 40 & 100 & 40 & 0.5581 & $29,897.3$ & 4.09 \\
\hline 13 & 40 & 100 & 60 & 0.4167 & $29,841.5$ & 3.90 \\
\hline 14 & 100 & 50 & 20 & 0.4206 & $29,828.7$ & 3.85 \\
\hline 15 & 100 & 50 & 40 & 0.6667 & $30,076.3$ & 4.72 \\
\hline 16 & 100 & 50 & 60 & 0.4333 & $29,837.4$ & 3.88 \\
\hline 17 & 100 & 100 & 20 & 0.4909 & $29,859.4$ & 3.96 \\
\hline 18 & 100 & 100 & 40 & 0.5333 & $29,888.2$ & 4.06 \\
\hline 19 & 100 & 100 & 60 & 0.5500 & $29,952.2$ & 4.28 \\
\hline
\end{tabular}

If we compute the average values of the variable avg.time-best for $d 2103$ and for $f l 3795$, we notice that the first is 47.09 and the second is 38.34 . This translates into larger execution times until the best solution is found (on average) for $d 2103$ than for $f l 3795$, although the number of nodes is smaller for the first instance. The explanation for this has to be searched into the structure of the instances. If we characterize the two instances by their order parameter $O P$, defined in [84] as the standard deviation of the distance matrix divided by its average value, we have $O P=0.4866$ for $d 2103$ and $O P=0.5393$ for $f l 3795$.

For the original MMAS, recovery speed $\in[0.3721,0.725]$ (see file fl3795), while for the applications corresponding to data in Table 4 (with ampl $=40$ ), recovery speed $\in[0.4167,0.7027]$. In this case, no value is smaller than the minimum recovery speed for MMAS with ampl $=0$. For this data set, the metric shows that $\operatorname{MMAS}(*, * *, 40)$ perform even better than the original implementation MMAS.

The results above allow us to qualitatively assess the resilience of the adapted MMAS applications as High [34].

Linear regression models for the metrics average-best and recovery speed follow in Tables 5 and 6.

Table 5. Regression model for average-best for fl3795.

\begin{tabular}{|c|c|c|c|c|}
\hline \multicolumn{5}{|c|}{ Dependent Variable: average-best } \\
\hline \multicolumn{5}{|c|}{ Method: Least Squares } \\
\hline \multicolumn{5}{|c|}{ Residuals: } \\
\hline Min & $1 Q$ & Median & $3 Q$ & Max \\
\hline-105.583 & -34.775 & -1.943 & 27.548 & 185.639 \\
\hline \multicolumn{5}{|l|}{ Coefficients: } \\
\hline & Estimate & Std. Error & $\mathrm{t}$ value & $\operatorname{Pr}(>|t|)$ \\
\hline (Intercept) & $2.976 \times 10^{+04}$ & $2.666 \times 10^{+01}$ & 1116.149 & $<2 \times 10^{-16 * * *}$ \\
\hline p_nod_ch & $2.951 \times 10^{-02}$ & $1.655 \times 10^{-01}$ & 0.178 & 0.859 \\
\hline p_inten & $1.722 \times 10^{-01}$ & $2.250 \times 10^{-01}$ & 0.765 & 0.446 \\
\hline epoch & $5.094 \times 10^{-01}$ & $3.445 \times 10^{-01}$ & 1.479 & 0.143 \\
\hline ampl10\% & $-1.551 \times 10^{+01}$ & $1.779 \times 10^{+01}$ & -0.872 & 0.386 \\
\hline $\operatorname{ampl} 20 \%$ & $1.003 \times 10^{+02}$ & $1.779 \times 10^{+01}$ & 5.640 & $2.35 \times 10^{-07 * * *}$ \\
\hline ampl30\% & $1.143 \times 10^{+02}$ & $1.779 \times 10^{+01}$ & 6.426 & $8.13 \times 10^{-09 * * *}$ \\
\hline ampl40\% & $9.683 \times 10^{+01}$ & $1.779 \times 10^{+01}$ & 5.442 & $5.34 \times 10^{-07 * * *}$ \\
\hline \multicolumn{5}{|c|}{ Signif. codes: $0^{\star * * * \prime} 0.001^{\prime * * \prime} 0.01^{\prime * \prime} 0.05^{\prime} \prime^{\prime} 0.1^{\prime \prime} 1$} \\
\hline \multicolumn{5}{|c|}{ Residual standard error: 53.38 on 82 degrees of freedom } \\
\hline \multicolumn{5}{|c|}{ Multiple R-squared: 0.5471, Adjusted R-squared: 0.5084} \\
\hline \multicolumn{5}{|c|}{ F-statistic: 14.15 on 7 and $82 \mathrm{DF}, p$-value: $6.583 \times 10^{-12}$} \\
\hline
\end{tabular}


Table 6. Regression model for recovery speed, instance $f l 3795$.

\begin{tabular}{|c|c|c|c|c|}
\hline \multicolumn{5}{|c|}{ Dependent Variable: recovery speed } \\
\hline \multicolumn{5}{|c|}{ Method: Least Squares } \\
\hline \multicolumn{5}{|l|}{ Residuals: } \\
\hline Min & $1 Q$ & Median & $3 Q$ & Max \\
\hline-10.991 & -3.594 & -0.893 & 1.503 & 112.522 \\
\hline \multicolumn{5}{|l|}{ Coefficients: } \\
\hline & Estimate & Std. Error & $\mathrm{t}$ value & $\operatorname{Pr}(>|t|)$ \\
\hline (Intercept) & -2.52477 & 6.55924 & -0.385 & 0.701 \\
\hline p_nod_ch & 0.05625 & 0.04071 & 1.382 & 0.171 \\
\hline p_inten & 0.05549 & 0.05536 & 1.002 & 0.319 \\
\hline epoch & -0.10271 & 0.08475 & -1.212 & 0.229 \\
\hline ampl10\% & -0.04463 & 4.37657 & -0.010 & 0.992 \\
\hline ampl $20 \%$ & 0.01811 & 4.37657 & 0.004 & 0.997 \\
\hline ampl30\% & 0.10873 & 4.37657 & 0.025 & 0.980 \\
\hline ampl40\% & 6.98307 & 4.37657 & 1.596 & 0.114 \\
\hline \multicolumn{5}{|c|}{ Signif. codes: $0^{* * * \prime} 0.001^{\star * * \prime} 0.01^{\star * \prime} 0.05^{\prime} ?^{\prime} 0.1^{\prime \prime} 1$} \\
\hline \multicolumn{5}{|c|}{ Residual standard error: 13.13 on 82 degrees of freedom } \\
\hline \multicolumn{5}{|c|}{ Multiple R-squared: 0.09325, Adjusted R-squared: 0.01584} \\
\hline \multicolumn{5}{|c|}{ F-statistic: 1.205 on 7 and $82 \mathrm{DF}, p$-value: 0.3096} \\
\hline
\end{tabular}

In Table 5, the adjusted R-Squared index shows that the linear regression model is good in the case of this instance, but knowing epoch, p_nod_ch, p_inten, and ampl, we could only explain $50.84 \%$ of the variance in average-best. ampl10\% is negative correlated to $\mathrm{H}$. Other independents are all positive correlated to $\mathrm{H}$.

The $p$ values show that ampl20\%, ampl30\%, ampl40\% are statistically significant in the model. Again, the behavior of the adapted MMAS differs from the case of $d 2013$. On fl3795, the quality of the solutions provided by our implementation is influenced (mostly) by epoch and ampl.

The adjusted R-Squared index shows that, in this case, there is no linear relation between the factors epoch, $p \_n o d \_c h, p \_i n t e n$, ampl and recovery speed. Again, the result differs from the correspondent one for $d 2103$.

The plot in Figure 2 presents the intercorrelations between the path-performance metrics average-best, stddev-best, best try and worst try and displays the distribution of each of them on the diagonal. On the bottom of the diagonal, the bivariate scatter plots with a fitted line are displayed. The value of the correlation and the significance level appear as stars on the top of the diagonal. Each significance level is associated to a symbol: $p$-values $(0,0.001,0.01,0.05,0.1,1)$ have the corresponding symbols $\left(* * *{ }^{\prime \prime},{ }^{(* * \prime},,^{* \prime \prime}, \prime^{\prime},,^{\prime}\right)$.

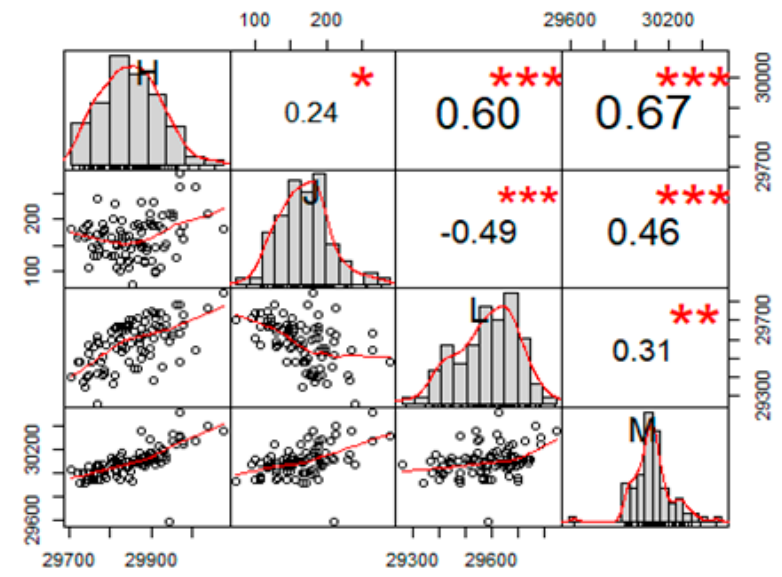

Figure 2. Intercorrelations between the path-performance metricsaverage-best, stddev-best, best try and worst try for fl3795. 


\subsection{Discussion on Behaviour of Adapted MMAS with TSP Instance rl1889}

The optimum for TSP instance rl1889 is 316,536. As data in Table 7 (except line no. 17) display, the relative error for the 18 variants of MMAS with ampl $=40$ is placed in the interval [0.49, 0.69], corresponding to an absolute error (distance-to-optimum in the file rl1889) in the interval [1565.4, 2184.6]. For this instance, the relative error is very small also, as it is for $d 2103$. We note that the minimum value for the relative-error is also obtained for MMAS with ampl=10 $(0.49$, in line 17$)$, and that this value corresponds to a smaller absolute error of 1543.8 .

Table 7. Parameters and metrics for $r l 1889$, with $a m p l=40$.

\begin{tabular}{crrrrrr}
\hline Line & p_nod_ch & p_inten & epoch & recovery speed & average-best & relative-error \\
\hline 1 & 20 & 50 & 20 & 0.8794 & $318,209.4$ & 0.53 \\
2 & 20 & 50 & 40 & 1.4754 & $318,245.4$ & 0.54 \\
3 & 20 & 50 & 60 & 1.5067 & $318,281.4$ & 0.55 \\
4 & 20 & 100 & 20 & 0.7461 & $318,173.4$ & 0.52 \\
5 & 20 & 100 & 40 & 1.4173 & $318,605.4$ & 0.65 \\
6 & 20 & 100 & 60 & 1.778 & $318,641.4$ & 0.67 \\
7 & 40 & 50 & 20 & 0.641 & $318,713.4$ & 0.69 \\
8 & 40 & 50 & 20 & 0.9652 & $318,720.6$ & 0.69 \\
9 & 40 & 50 & 40 & 1.8023 & $318,677.4$ & 0.68 \\
10 & 40 & 50 & 60 & 1.9559 & $318,317.4$ & 0.56 \\
11 & 40 & 100 & 20 & 0.8115 & $318,137.4$ & 0.51 \\
12 & 40 & 100 & 40 & 1.7883 & $318,353.4$ & 0.57 \\
13 & 40 & 100 & 60 & 2.3153 & $318,569.4$ & 0.64 \\
14 & 100 & 50 & 20 & 0.8474 & $318,461.4$ & 0.61 \\
15 & 100 & 50 & 40 & 1.3854 & $318,497.4$ & 0.62 \\
16 & 100 & 50 & 60 & 2.1518 & $318,533.4$ & 0.63 \\
17 & 100 & 100 & 20 & 0.9206 & $318,079.8$ & 0.491 \\
18 & 100 & 100 & 20 & 0.9728 & $318,101.4$ & 0.49 \\
19 & 100 & 100 & 40 & 1.2885 & $318,389.4$ & 0.59 \\
20 & 100 & 100 & 60 & 2.4444 & $318,425.4$ & 0.60 \\
\hline & & & 1 & Value for $a m p l=10$. & &
\end{tabular}

For the original MMAS, recovery speed $\in[0.7864,2.5652]$ (see file $r(1889)$, while for the applications corresponding to data in Table 7 (with ampl $=40$ ), recovery speed $\in[0.641,2.444]$, with only two values smaller than the minimum recovery speed for MMAS with $a m p l=0$. For this data set, the metric shows that $\operatorname{MMAS}(*, *, *, 40)$ continue to behave very well and deliver quality solutions.

The results above allow us to qualitatively assess the resilience of the adapted MMAS applications as High [34] for this instance, too.

Linear regression model for the metric average-best follows in Table 8.

Table 8. Regression model for average-best, instance rl1889.

\begin{tabular}{|c|c|c|c|c|}
\hline \multicolumn{5}{|c|}{ Dependent Variable: average-best } \\
\hline \multicolumn{5}{|c|}{ Method: Least Squares } \\
\hline \multicolumn{5}{|c|}{ Residuals: } \\
\hline Min & $1 \mathrm{Q}$ & Median & $3 \mathrm{Q}$ & $\operatorname{Max}$ \\
\hline-231.23 & -156.69 & -8.88 & 113.31 & 359.31 \\
\hline \multicolumn{5}{|l|}{ Coefficients: } \\
\hline & Estimate & Std. Error & $\mathrm{t}$ value & $\operatorname{Pr}(>|t|)$ \\
\hline (Intercept) & $3.183 \times 10^{+05}$ & $9.001 \times 10^{+01}$ & 3536.748 & $<2 \times 10^{-16 * * *}$ \\
\hline p_nod_ch & $1.731 \times 10^{-01}$ & $5.587 \times 10^{-01}$ & 0.310 & 0.7575 \\
\hline p_inten & $-1.200 \times 10^{+00}$ & $7.597 \times 10^{-01}$ & -1.580 & 0.1180 \\
\hline epoch & $4.050 \times 10^{+00}$ & $1.163 \times 10^{+00}$ & 3.482 & $0.0008^{* * *}$ \\
\hline ampl10\% & $-2.880 \times 10^{+01}$ & $6.006 \times 10^{+01}$ & -0.480 & 0.6328 \\
\hline ampl20\% & $-2.160 \times 10^{+01}$ & $6.006 \times 10^{+01}$ & -0.360 & 0.7200 \\
\hline ampl30\% & $-1.440 \times 10^{+01}$ & $6.006 \times 10^{+01}$ & -0.240 & 0.8111 \\
\hline ampl40\% & $-7.200 \times 10^{+00}$ & $6.006 \times 10^{+01}$ & -0.120 & 0.9049 \\
\hline \multicolumn{5}{|c|}{ 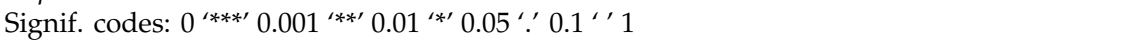 } \\
\hline \multicolumn{5}{|c|}{ Residual standard error: $108.2 \mathrm{on} 82$ degrees of freedom } \\
\hline \multicolumn{5}{|c|}{ Multiple R-squared: 0.1547, Adjusted R-squared: 0.08253} \\
\hline \multicolumn{5}{|c|}{ F-statistic: 2.144 on 7 and $82 \mathrm{DF}, p$-value: 0.04789} \\
\hline
\end{tabular}


Considering the adjusted R-Squared index we conclude that the linear regression model does not explain the average-best dependence of epoch, p_nod_ch, p_inten, ampl. As in the case of $d 2103$, the implementation performs very well for every combination of parameters.

For the metric recover speed, the linear regression model is presented in Table 9.

Table 9. Regression model for recovery speed, instance $r l 1889$.

\begin{tabular}{|c|c|c|c|c|}
\hline \multicolumn{5}{|c|}{ Dependent Variable: recovery speed } \\
\hline \multicolumn{5}{|c|}{ Method: Least Squares } \\
\hline \multicolumn{5}{|l|}{ Residuals: } \\
\hline Min & $1 Q$ & Median & $3 Q$ & $\operatorname{Max}$ \\
\hline-0.59491 & -0.12230 & -0.00215 & 0.12432 & 0.56298 \\
\hline \multicolumn{5}{|l|}{ Coefficients: } \\
\hline & Estimate & Std. Error & $\mathrm{t}$ value & $\operatorname{Pr}(>|t|)$ \\
\hline (Intercept) & 0.2704582 & 0.1120153 & 2.414 & 0.018 * \\
\hline p_nod_ch & 0.0010162 & 0.0006953 & 1.462 & 0.148 \\
\hline p_inten & 0.0007799 & 0.0009454 & 0.825 & 0.412 \\
\hline epoch & 0.0275353 & 0.0014473 & 19.025 & $<2 \times 10^{-16 * * *}$ \\
\hline ampl10\% & -0.0380889 & 0.0747408 & -0.510 & 0.612 \\
\hline ampl $20 \%$ & -0.0471611 & 0.0747408 & -0.631 & 0.530 \\
\hline ampl30\% & -0.0201778 & 0.0747408 & -0.270 & 0.788 \\
\hline ampl40\% & -0.0285889 & 0.0747408 & -0.383 & 0.703 \\
\hline \multicolumn{5}{|c|}{ Signif. codes: $0^{\star * * * \prime} 0.001^{* * * \prime} 0.01^{* *} 0.05^{\prime} .^{\prime} 0.1^{\prime \prime} 1$} \\
\hline \multicolumn{5}{|c|}{ Residual standard error: 0.2242 on 82 degrees of freedom } \\
\hline \multicolumn{5}{|c|}{ Multiple R-squared: 0.8166, Adjusted R-squared: 0.801} \\
\hline \multicolumn{5}{|c|}{ F-statistic: 52.17 on 7 and $82 \mathrm{DF}, p$-value: $<2.2 \times 10^{-16}$} \\
\hline
\end{tabular}

This model indicates a negative correlation of the metric recovery speed with ampl and positive correlations with $p \_n o d-c h, p \_i n t e n$ and epoch. As for the case of instance $d 2103$, only the factors epoch and ampl are significant in the model.

In Figure 3 we chose to display the same type of correlogram as in Figure 1, in order to compare the intercorrelations of other time-performance metrics for $d 2103$ and rl1889.

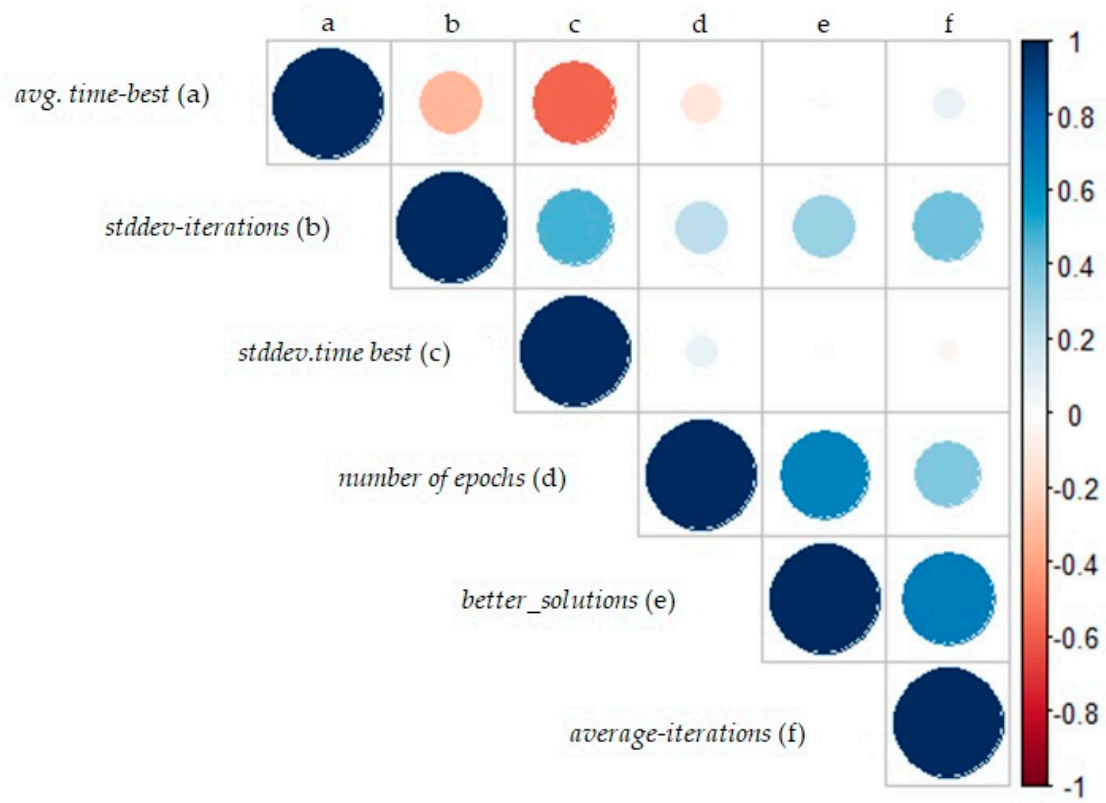

Figure 3. Correlogram of the time-performance metrics avg.time-best, stddev-best, stddev.time best, average-iterations, better_solutions and number of epochs for rl1889. 
We notice the same types of correlations for $d 2103$ as for $r 11889$, except for average-iterations and stddev.time best, for which the correlation is positive for $d 2103$ and negative for $r l 1889$. For $r l 1889$, the order parameter [84] is $\mathrm{OP}=0.5129$.

\section{Discussion}

The statistical analysis of data recorded for two of the studied TSP instances ( $d 2103$ and $r 11889$ ) reveals that the adapted MMAS implementation is highly resilient: both the delivered solutions and the time-performance metrics show that the ant algorithms perform very well when distortions appear. For these two instances, under the settings presented in Section 4.2, the quality of the solutions returned by MMAS(epoch, p_nod_ch, p_inten, ampl) do not depend on the four factors, while the recovery speed depends on epoch and ampl. These facts, supported by the statistical analysis of the experimental data, allow us to conclude that the applications are highly resilient. For the other TSP instance, fl3795, the adapted MMAS also proves to be resilient, but with a different behavior pattern: the quality of the solutions returned is slightly influenced by epoch and ampl, while the recovery speed does not depend on the four factors. The answer to the question "Why does this happen?" could be searched among multiple causes, but two are immediate: the number of nodes and the instances of structures. These will drive our next studies: one focused on larger instances and another based on the structural features [85] of TSP instances. The nondeterministic nature of MMAS can also suggest that research on another output data set, for the same instances, is worth being investigated.

Our previous research with ACO [86,87] gave us some insight on the ant algorithms behavior under different circumstances. When referring to the resilience attributes synthesized in [34], we notice that all of them are representative for this class of algorithms. A brief enumeration of resilience attributes (and their translation to ACO and the artificial ants) include: interconnection among the agents (artificial ants communicate, which leads to system flexibility); redundancy of units (artificial ants perform the same simple tasks, but the performance of the system emerges from their interaction); diversity and variety in resilient systems, which means that there are different parts and a variety of ways of functioning (all these features have been implemented in ACO); the diversity of approaches, which is about generating various views on a situation (also possible in ACO). Moreover, parallelism and distributiveness (implicit in ACO, with no central command and no single point of failure) and handling of increased workloads (easily achievable with ACO), dynamic learning (the pheromone updating mechanism) are important attributes. Finally, self-organization lies in the natural model that inspired ACO and further developments of this heuristic approach.

When optimization problems arise in practice, the users need to have confidence in the quality of the solutions provided by their applications. There are many situations when things do not go "by the book". Sometimes unexpected events happen and change the graph representation of a problem, or make some data unavailable. Other demanding situations can emerge when communication is interrupted, data are imprecise or mistaken, or even human errors appear. In such cases, if applications are resilient, these manage to provide solutions which are sufficiently close to those provided in normal conditions. Ant algorithms are systems that behave in a manner that is widely accepted by researchers and practitioners as resilient. One goal for our future work would be the design of ant algorithms that embed other types of intelligence and are able to learn and adapt. This falls into line with the innovative proposal of Florio [88]: “ ... we need to look at the nature and change the paradigm of resilience from «being at work while staying the same» to «being at work while getting better», namely becoming a better system or being".

Author Contributions: Conceptualization, methodology, writing-original draft preparation, writing-review and editing, E.N., G.C.C., L.B.I.; software, G.C.C.; formal analysis, visualization, Y.H.; investigation, E.N., G.C.C., L.B.I.; project administration, funding acquisition, E.N. All authors have read and agreed to the published version of the manuscript. 
Funding: The research performed by E.N., G.C.C., and the APC were funded by the Ministry of Education and Research, through the National Council for the Financing of Higher Education, Romania, grant number CNFIS-FDI-2019-0453.

Conflicts of Interest: The authors declare no conflict of interest. The funders had no role in the design of the study; in the collection, analyses, or interpretation of data; in the writing of the manuscript, or in the decision to publish the results.

\section{References}

1. Fiskel, J. Designing resilient, sustainable systems. Environ. Sci. Technol. 2003, 37, 5330-5339. [CrossRef]

2. Arthur, W. Complexity and the economy. Science 1999, 284, 107-109. [CrossRef] [PubMed]

3. Starr, R.; Newfrock, J.; Delurey, M. Enterprise resilience: Managing risk in the network economy. Strategy Bus. 2004, 30, 1-10. Available online: https://www.strategy-business.com/article/8375?gko=9ee65 (accessed on 19 March 2020).

4. Folke, C.; Carpenter, S.; Elmquist, T.; Gunderson, L.; Holling, C.; Walker, B. Resilience and sustainable development: Building adaptive capacity in a world of transformations. Ambio 2002, 31, 437-440. [CrossRef]

5. Lal, R. Sustainable land use systems and soil resilience. In Soil Resilience and Sustainable Land Use; Greenland, D., Szabolcs, I., Eds.; CAB International: Wallingford, UK, 1994; pp. 41-67.

6. Gunderson, L.H. Ecological Resilience-In Theory and Application. Annu. Rev. Ecol. S 2000, 31, 425-439. [CrossRef]

7. Standish, R.J.; Hobbs, R.J.; Mayfield, M.M.; Bestelmeyer, B.T.; Suding, K.N.; Battaglia, L.L.; Eviner, V.; Hawkes, C.V.; Temperton, V.M.; Cramer, V.A.; et al. Resilience in ecology: Abstraction, distraction, or where the action is? Biol. Conserv. 2014, 177, 43-51. [CrossRef]

8. Bonnano, G. Loss, trauma, and human resilience: Have we underestimated the human capacity to thrive after extremely aversive events? Am. Psychol. 2004, 59, 20-28. [CrossRef]

9. Rutter, M. Implications of Resilience Concepts for Scientific Understanding. Ann. N.Y. Acad. Sci. 2006. [CrossRef]

10. Beery, A.K.; Kaufer, D. Stress, social behaviour, and resilience: Insights from rodents. Neurobiol. Stress 2015, 1, 116-127. [CrossRef]

11. Adger, W. Social and ecological resilience: Are they related? Progr. Hum. Geogr. 2000, 24, 347-364. [CrossRef]

12. Calloway, D.E.; Newman, M.E.J.; Strogatz, S.H.; Watts, D.J. Network Robustness and Fragility: Percolation on Random Graphs. Phys. Rev. Lett. 2000, 85, 5468-5471. [CrossRef] [PubMed]

13. Adler, C.O.; Dagli, C.H. Study of the Use of a Genetic Algorithm to Improve Networked System-of-Systems Resilience. Procedia Comput. Sci. 2014, 36, 49-56. [CrossRef]

14. Sha, Z.; Panchal, J. Towards the design of complex evolving networks with high robustness and resilience. Procedia Comput. Sci. 2013, 16, 522-531. [CrossRef]

15. Trivedi, K.S.; Kim, D.D.; Ghosh, R. Resilience in computer systems and networks. In Proceedings of the 2009 International Conference on Computer-Aided Design (ICCAD'09), San Jose, CA, USA, 2-5 November 2009. [CrossRef]

16. Matni, N.; Leong, Y.P.; Wang, Y.S.; You, S.; Horowitz, M.B.; Doyle, J.C. Resilience in Large Scale Distributed Systems. Procedia Comput. Sci. 2014, 28, 285-293. [CrossRef]

17. Yu, D.J.; Rao, P.S.; Klinkhamer, C.; Krueger, E.H.; Sangwan, N.; Sung, K. Aligning Different Schools of Thought on Resilience of Complex Systems and Networks. IRGC Resource Guide on Resilience v29-07-2016 2016, Lausanne: EPFL International Risk Governance Center. Available online: https://www.irgc.org/ riskgovernance/resilience/ (accessed on 19 March 2020).

18. Ford, R.; Carvalho, M.; Mayron, L.; Bishop, M. Towards Metrics for Cyber Resilience. In Proceedings of the 21st EICAR Annual Conference Proceedings, Lisbon, Portugal, 7-8 May 2012; pp. 151-159.

19. Omer, A.; Mostashari, A.; Lindemann, U. Resilience Analysis of Soft Infrastructure Systems. Procedia Comput. Sci. 2014, 28, 565-574. [CrossRef]

20. Cutter, S.L.; Barnes, L.; Berry, M.; Evans, E.; Tate, E.; Webb, J. A place-based model for understanding community resilience to natural disasters. Glob. Environ. Change 2008, 18, 598-606. [CrossRef]

21. Jordan, E.; Javernick-Will, A. Measuring Community Resilience and Recovery: A Content Analysis of Indicators. Constr. Res. Congr. 2012, 2190-2199. [CrossRef] 
22. Scott, J. Architecting Resilient Systems: Accident Avoidance and Survival and Recovery from Disruptions; Wiley Series in Systems Engineering and Management; John Wiley and Sons: Hoboken, NJ, USA, 2010.

23. MacAskill, K.; Guthrie, P. Multiple interpretations of resilience in disaster risk management. Procedia Econ. Financ. 2014, 18, 667-674. [CrossRef]

24. Chien, A.; Baaji, P.; Beckman, P.; Dun, N.; Fang, A.; Fujita, H.; Iskra, K.; Rubenstein, Z.; Zheng, Z.; Schreiber, R.; et al. Versioned Distributed Arrays for Resilience in Scientific Applications: Global View Resilience. Procedia Comput. Sci. 2015, 51, 29-38. [CrossRef]

25. Wright, C.; Kiparoglou, V.; Williams, M.; Hilton, J. A Framework for Resilience Thinking. Procedia Comput. Sci. 2012, 8, 45-52. [CrossRef]

26. Bruneau, M.; Reinhorn, A. Overview of the Resilience Concept. In Proceedings of the 8th US National Conference on Earthquake Engineering, San Francisco, CA, USA, 18-22 April 2006; p. 2040.

27. Proag, V. The concept of vulnerability and resilience. Procedia Econ. Financ. 2014, 18, 369-376. [CrossRef]

28. Bahill, T.A.; Botta, R. Fundamental Principles of Good Systems Design. Eng. Manag. J. 2008, 20, 9-17. [CrossRef]

29. Sols, A. Increasing Systems Resilience through the Implementation of a Proactive Technology Refreshment Program. Procedia Comput. Sci. 2014, 28, 26-33. [CrossRef]

30. Alsberg, A.P.; Day, J.D. A principle for resilient sharing of distributed resources. In Proceedings of the ICSE '76: 2nd International Conference on Software Engineering, San Francisco, CA, USA, 13-15 October 1976; Yeh, R.T., Ramamoorthy, C.V., Eds.; IEEE Computer Society Press: Washington, DC, USA, 1976; pp. 562-570.

31. Avizienis, A.; Laprie, J.C.; Randell, B.; Landwehr, C. Basic Concepts and Taxonomy of Dependable and Secure Computing. IEEE Trans. Dependable Secure Comput. 2004, 1, 11-33. [CrossRef]

32. Axelrod, C.W. Investing in Software Resiliency. J. DefenseSoftw. Eng. 2009, 22, 20-25.

33. Marcus, E.; Stern, H. Blueprints for High Availability: Designing Resilient Distributed Systems; John Wiley \& Sons: New York, NY, USA, 2000.

34. Chandra, A. Synergy between Biology and Systems Resilience. Master's Thesis, Missouri University of Science and Technology, Rolla, MO, USA, 2010. Available online: https://scholarsmine.mst.edu/masters_theses/6728 (accessed on 19 March 2020).

35. Duijnhoven, H.; Neef, M. Framing Resilience. From a model-based approach to management process. Procedia Econ. Financ. 2014, 18, 425-430. [CrossRef]

36. Proag, V. Assessing and measuring resilience. Procedia Econ. Financ. 2014, 18, 222-229. [CrossRef]

37. Li, L.; Peng, H.; Kurths, J.; Yang, Y.; Schellnhuber, H.J. Chaos-order transition in foraging behavior of ants. Proc. Natl. Acad. Sci. USA 2014, 111, 8392-8397. [CrossRef]

38. Chittka, L.; Muller, H. Learning, specialization, efficiency and task allocation in social insects. Commun. Integr. Biol. 2009, 2, 151-154. [CrossRef]

39. Morgan, E.D. Trail pheromones of ants. Physiol. Entomol. 2009, 34, 1-17. [CrossRef]

40. Dorigo, M.; Maniezzo, V.; Colorni, A. The Ant System: Optimization by a colony of cooperating agents. IEEE Trans. Syst. Man Cybern. Part B 1996, 26, 1-13. [CrossRef] [PubMed]

41. Dorigo, M.; Gambardella, L.M. Ant Colonies for the Traveling Salesman Problem; Technical Report TR/IRIDIA/1996-3; Université Libre de Bruxelles: Brussels, Belgium, 1996.

42. Dorigo, M.; Di Caro, G. The Ant Colony Optimization Meta-Heuristic. New Ideas in Optimization; McGraw-Hill: New York, NY, USA, 1999.

43. Applegate, D.L.; Bixby, R.E.; Chvátal, V.; Cook, W.J. The Traveling Salesman Problem: A Computational Study. In Princeton Series in Applied Mathematics; Princeton University Press: Princeton, NJ, USA, 2011.

44. Cook, W.J. Pursuit of the Traveling Salesman: Mathematics at the Limits of Computation; Princeton University Press: Princeton, NJ, USA, 2012.

45. Dantzig, G.B.; Fulkerson, R.; Johnson, S.M. Solution of a Large-Scale Traveling Salesman Problem. Oper. Res. 1954, 2, 393-410. [CrossRef]

46. Karp, R.M. Reducibility among Combinatorial Problems. In Complexity of Computer Computations; The IBM Research, Symposia; Miller, R.E., Thatcher, J.W., Eds.; Plenum Press: New York, NY, USA, 1972; pp. 85-103.

47. Woeginger, G.J. Exact Algorithms for NP-Hard Problems: A Survey. In Combinatorial Optimization-Eureka, You Shrink! Lecture Notes in Computer, Science; Jünger, M., Reinelt, G., Rinaldi, G., Eds.; Springer: Berlin/Heidelberg, Germany, 2003; Volume 2570, pp. 185-207. [CrossRef] 
48. Held, M.; Karp, R.M. The Traveling-Salesman Problem and Minimum Spanning Trees. Oper. Res. 1970, 18, 1138-1162. [CrossRef]

49. Held, M.; Karp, R.M. The Traveling-Salesman Problem and Minimum Spanning Trees: Part II. Math. Program. 1971, 1, 6-25. [CrossRef]

50. Concorde TSP Solver. Available online: http://www.math.uwaterloo.ca/tsp/concorde/ (accessed on 19 March 2020).

51. Rojanasoonthon, S.; Bard, S.F.; Reddy, S.D. Algorithms for parallel machine scheduling: A case study of the tracking and data relay satellite system. J. Oper. Res. Soc. 2003, 54, 806-821. [CrossRef]

52. Ratliff, H.D.; Rosenthal, A.S. Order-Picking in a Rectangular Warehouse: A Solvable Case for the Travelling Salesman Problem. Oper. Res. 1983, 31, 507-521. [CrossRef]

53. Grötschel, M.; Jünger, M.; Reinelt, G. Optimal Control of Plotting and Drilling Machines: A Case Study. Math. Method Oper. Res. 1991, 35, 61-84. [CrossRef]

54. Lee, J.Y.; Shin, S.Y.; Park, T.H.; Zhang, B.T. Solving traveling salesman problems with DNA molecules encoding numerical values. Biosystems 2004, 78, 39-47. [CrossRef]

55. Grover, L. Local search and the local structure of NP-complete problems. Oper. Res. Lett. 1992, 12, $235-243$. [CrossRef]

56. Codenotti, B.; Margara, L. Traveling Salesman Problem and Local Search. Appl. Math. Lett. 1992, 5, 69-71. [CrossRef]

57. Gamboa, D.; Rego, C.; Glover, F. Data Structures and Ejection Chains for Solving Large Scale Traveling Salesman Problems. Eur. J. Oper. Res. 2005, 160, 154-171. [CrossRef]

58. Lin, S.; Kernighan, B.W. An Effective Heuristic Algorithm for the Traveling-Salesman Problem. Oper. Res. 1973, 21, 498-516. [CrossRef]

59. Applegate, D.; Cook, W.J.; Rohe, A. Chained Lin-Kernighan for large traveling salesman problems. INFORMS J. Comput. 2003, 15, 82-92. [CrossRef]

60. Kirkpatrick, S.; Gelatt, C.D., Jr.; Vecchi, M.P. Optimization by Simulated Annealing. Science 1983, 220, 671-680. [CrossRef] [PubMed]

61. Zhou, A.H.; Zhu, L.P.; Hu, B.; Deng, S.; Song, Y.; Qiu, H.; Pan, S. Traveling-Salesman-Problem Algorithm Based on Simulated Annealing and Gene-Expression Programming. Information 2019, 10, 7. [CrossRef]

62. Scholz, J. Genetic Algorithms and the Traveling Salesman Problem. A Historical Review. In Proceedings of the MInf Seminar at the Dept. of Computer Science of the Hamburg University of Applied Sciences, Winter 2017/2018, Hamburg, Germany. Available online: https://arxiv.org/pdf/1901.05737.pdf (accessed on 19 March 2020).

63. Dorigo, M.; Birattari, M.; Stutzle, T. Ant Colony Optimization. IEEE Comput. Intell. Mag. 2006, 1, $28-39$. [CrossRef]

64. Stützle, T. ACOTSP Software. 2004. Available online: https://www.swmath.org/software/11237 (accessed on 19 March 2020).

65. Costa, Y.J.; Castaño, N.; Betancur, J.F. Optimization based on Multi-type Ants for the Traveling Salesman Problem. In Proceedings of the 9th Computing Colombian Conference (9CCC), Pereira, Colombia, 3-5 September 2014; IEEE Press: New York, NY, USA, 2014; pp. 144-149.

66. Holzinger, A.; Plass, M.; Kickmeier-Rust, M.; Holzinger, K.; Crisan, G.C.; Pintea, C.M.; Palade, V. Interactive machine learning: Experimental evidence for the human in the algorithmic loop. Appl. Intell. 2019, 49, 2401-2414. [CrossRef]

67. Jovanovic, R.; Tuba, M. Ant Colony Optimization Algorithm with Pheromone Correction Strategy for the Minimum Connected Dominating Set Problem. Comput. Sci. Inf. Syst. 2013, 10, 133-149. [CrossRef]

68. Lopez-Ibáñez, M.; Stützle, T. An Analysis of Algorithmic Components for Multiobjective Ant Colony Optimization: A Case Study on the Biobjective TSP. In Lecture Notes in Computer Science; Artifical Evolution. EA; Collet, P., Monmarché, N., Legrand, P., Schoenauer, M., Lutton, E., Eds.; Springer: Berlin/Heidelberg, Germany, 2009; Volume 5975. [CrossRef]

69. López-Ibáñez, M.; Stützle, T. Automatically improving the anytime behaviour of optimisation algorithms. Eur. J. Oper. Res. 2014, 235, 569-582. [CrossRef] 
70. Dorigo, M. Optimization, Learning and Natural Algorithms. Ph.D. Thesis, Dipartimento di Elettronica, Politecnico di Milano, Milan, Italy, 1991. (In Italian)

71. Dorigo, M.; Maniezzo, V.; Colorni, A. Positive Feedback as a Search Strategy; Technical Report 91-016; Dipartimento di Elettronica, Politecnico di Milano: Milan, Italy, 1991.

72. Dorigo, M.; Stützle, T. The Ant Colony Optimization Metaheuristic: Algorithms, Applications, and Advances. In Handbook of Metaheuristics. International Series in Operations Research \& Management Science; Glover, F., Kochenberger, G.A., Eds.; Springer: Boston, MA, USA, 2003; Volume 57. [CrossRef]

73. Stützle, T.; Hoos, H.H. MAX-MIN Ant System. Future Gener. Comput. Syst. 2000, 16, 889-914. [CrossRef]

74. Data Files and Statistical Analysis Files for d2013, fl3795 and rl1889. Available online: http://cadredidactice. ub.ro/ceraselacrisan/2d-euclidean-tsp-instances/ (accessed on 1 March 2020).

75. Crişan, G.C.; Nechita, E.; Palade, V. Ant-Based System Analysis on the Traveling Salesman Problem under Real-World Settings. In Combinations of Intelligent Methods and Applications. Smart Innovation, Systems and Technologies; Hatzilygeroudis, I., Palade, V., Prentzas, J., Eds.; Springer: Berlin/Heidelberg, Germany, 2016; Volume 46, pp. 39-59. [CrossRef]

76. TSPLIB. Available online: http://comopt.ifi.uni-heidelberg.de/software/TSPLIB95/ (accessed on 1 March 2020).

77. Discrete and Combinatorial Optimization, Universität Heidelberg Institut für Informatik. Available online: http://comopt.ifi.uni-heidelberg.de/software/TSPLIB95/ (accessed on 19 March 2020).

78. Jünger, M.; Reinelt, G.; Rinaldi, G. The Traveling Salesman Problem. The Institute for Systems Analysis and Computer Science, Rome, Technical Report R375. 1994. Available online: http://www.iasi.cnr.it/reports/R375/ R375.pdf (accessed on 16 April 2020).

79. Johnson, D.S.; McGeoch, L.A. The Traveling Salesman Problem: A Case Study. In Local Search in Combinatorial Optimization; Aarts, E.H.L., Lenstra, J.K., Eds.; Princeton University Press: Princeton, NJ, USA; Oxford, UK, 2003; pp. 215-310. [CrossRef]

80. Birattari, M.; Dorigo, M. How to assess and report the performance of a stochastic algorithm on a benchmark problem: Mean or best result on a number of runs? Optim. Lett. 2007, 1, 309-311. [CrossRef]

81. Jackson, S. System Resilience: Capabilities, Culture and Infrastructure. In Proceedings of the INCOSE International Symposium, San Diego, CA, USA, 24-28 June 2007; Wiley Online Library, 2014; Volume 17, pp. 885-899. [CrossRef]

82. Tamvakis, P.; Xenidis, Y. Resilience in Transportation Systems. Procedia Soc. Behav. Sc. 2012, 48, 3441-3450. [CrossRef]

83. Crişan, G.C. Ant Algorithms in Artificial Intelligence. Ph.D. Thesis, Al.I. Cuza University of Iaşi, Iaşi, Romania, 2008.

84. Cheeseman, P.; Kanefsky, B.; Taylor, W.M. Where the really hard problems are. In Proceedings of the IJCAI'91: 12th International Joint Conference on Artificial Intelligence, Sydney, Australia, 24-30 August 1991; Morgan Kaufmann Publishers: Burlington, MA, USA, 1991; Volume 1, pp. 331-337. [CrossRef]

85. Nallaperuma, S.; Wagner, M.; Neumann, F. Analyzing the effects of instance features and algorithm parameters for Max-Min Ant System and the Traveling Salesperson Problem. Front. Robot AI 2015, 2. [CrossRef]

86. Czibula, G.; Crişan, G.C.; Pintea, C.M.; Czibula, I.G. Soft Computing Approaches on the Bandwidth Problem. Informatica 2013, 24, 169-180. [CrossRef]

87. Pintea, C.M.; Crişan, G.C.; Chira, C. Hybrid ant models with a transition policy for solving a complex problem. Logic. J. IGPL 2012, 20, 560-569. [CrossRef]

88. De Florio, V. Systems, Resilience, and Organization: Analogies and Points of Contact with Hierarchy Theory. In Proceedings of the 4th International Workshop on Computational Antifragility and Antifragile Engineering (ANTIFRAGILE 2017), Trieste, Italy, 3-6 July 2017; Available online: https://arxiv.org/abs/1411.0092 (accessed on 20 April 2020).

(C) 2020 by the authors. Licensee MDPI, Basel, Switzerland. This article is an open access article distributed under the terms and conditions of the Creative Commons Attribution (CC BY) license (http://creativecommons.org/licenses/by/4.0/). 